\title{
Grisel Syndrome in Adult Patients. Report of Two Cases and Review of the Literature
}

\author{
Karam Youssef, Shedid Daniel
}

Can. J. Neurol. Sci. 2009; 36: 109-113

Grisel's syndrome involves the subluxation of the atlantoaxial joint from inflammatory ligamentous laxity following an infectious process in the head or neck ${ }^{1}$. It rarely occurs in adults. In children, the treatment is first medical with the need for immobilization and sometimes internal fixation depending on the stability of C1-C2. In this paper we report two cases of Grisel syndrome in adults.

\section{CASE RePort}

\section{Case 1}

A 50-year-old female intravenous drug user presented with neck pain and electrical sensations in the upper and lower limbs on neck flexion (Lhermitte's sign). There was no history of head and neck trauma but she reported tonsilopharyngitis one to two weeks prior to consultation. Her physical examination was normal except for a positive Lhermitte's sign. Magnetic resonance imaging (MRI) of the cervical spine revealed a mass infiltrating the posterior subligamentous region of the anterior arch of $\mathrm{C} 1$, the odontoid and the body of $\mathrm{C} 2$ and compressing the bulbo-medullary junction (Figure 1). and an increase of the atlanto-dental interval (Figure 2). The CT-scan did not show any bony destruction or a rotatory subluxation (Figure 3 ). The patient received antibiotics with wide spectrum coverage and was stabilized in a Halo vest after closed reduction. The radiological follow-up revealed a decrease in the prevertebral collection as well as a decrease in the spinal cord compression (Figure 4 and 5).

After three months the patient fell and presented to the emergency room with loosening of the Halo. She was neurologically intact but the flexion cervical $\mathrm{x}$-ray revealed a C1$\mathrm{C} 2$ instability (Figure 6). The patient underwent a C1/C2 fusion, with C1 lateral mass screw and a pars screw (Figure 7). At her last follow-up (five months), the patient was doing very well and was pain-free.

\section{Case 2}

A 53-year-old alcoholic male was admitted to the service of gastroenterology for alteration of vigilance and gastro-intestinal bleeding. No neurological deficit was found. Blood workup showed elevated hepatic enzymes, electrolyte disturbances, anemia and leukocytosis. He also had positive hemoculture for gram negative rods but no septic focus was found. A meningitis was ruled out. Before doing a lumbar puncture, a brain MRI was done to rule out an abcess. This showed a peri-odontoidal infiltration with an increased atlanto-dental interval and retropharyngeal abcess (Figures 8 and 9). The investigation was

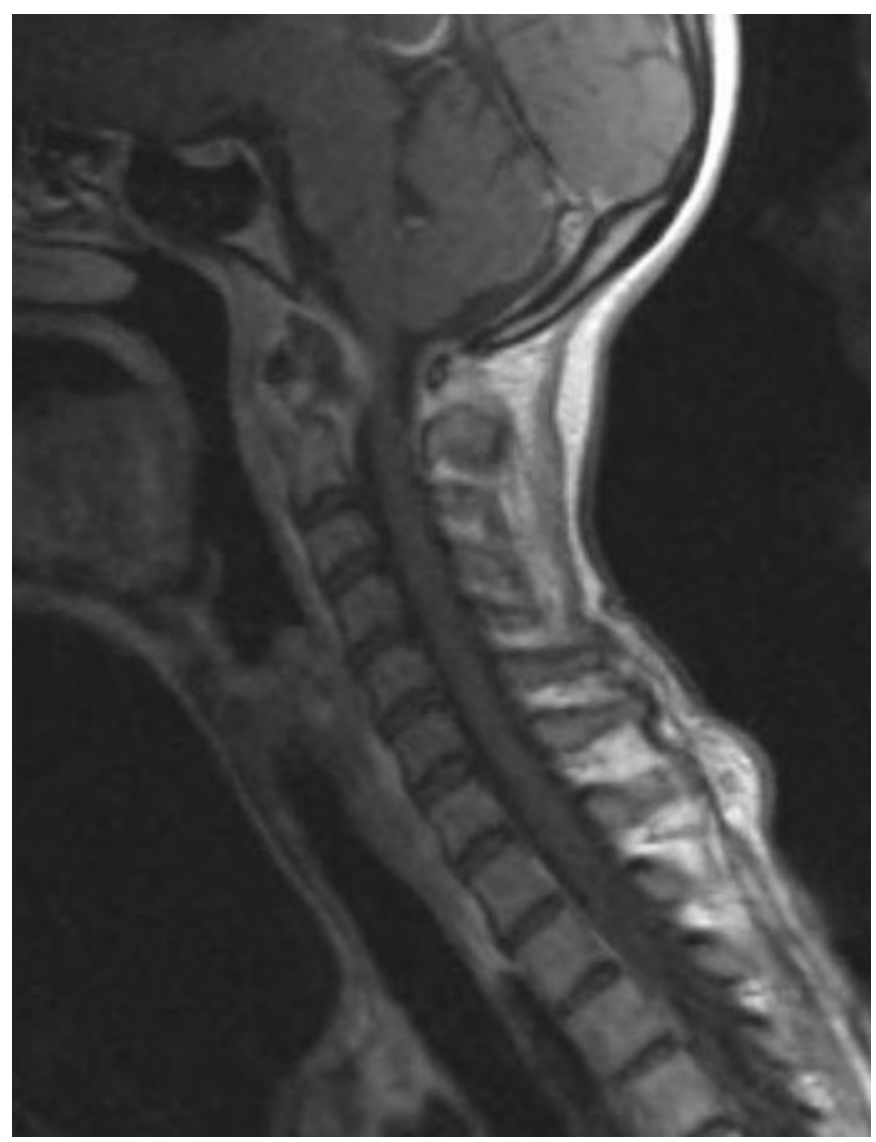

Figure 1: Sagital T1 contrast enhanced MRI Grisel's syndrome.

completed with plain films (Figure 10), CT-scan and MRI of the cervical spine. There was no bone decalcification nor rotatory subluxation. The patient was treated with antibiotics and stabilized clinically before operation. He then underwent a $\mathrm{C} 1 / \mathrm{C} 2$ fusion, with $\mathrm{C} 1$ lateral mass screw and a pars screw

From the Division of Neurosurgery, Spine Surgery Unit, Centre Hospitalier de l'Université de Montréal. Notre-Dame Hospital. Montréal, Québec, Canada. Received March 7, 2008. Final Revisions Submitted September 5, 2008 Correspondence to: Youssef Karam, Montréal University, 1690 Sherbrooke St. East, Montréal, Québec, H2L 1M5, Canada. 


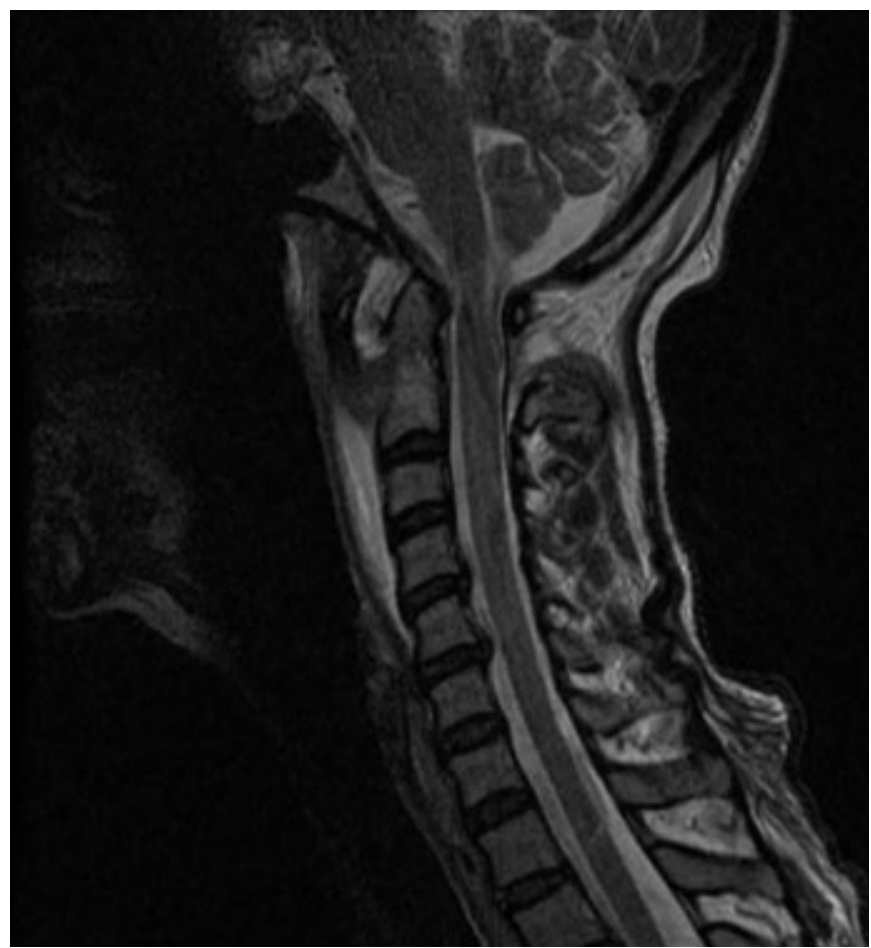

Figure 2: Sagittal T2 showing the increased ADI.

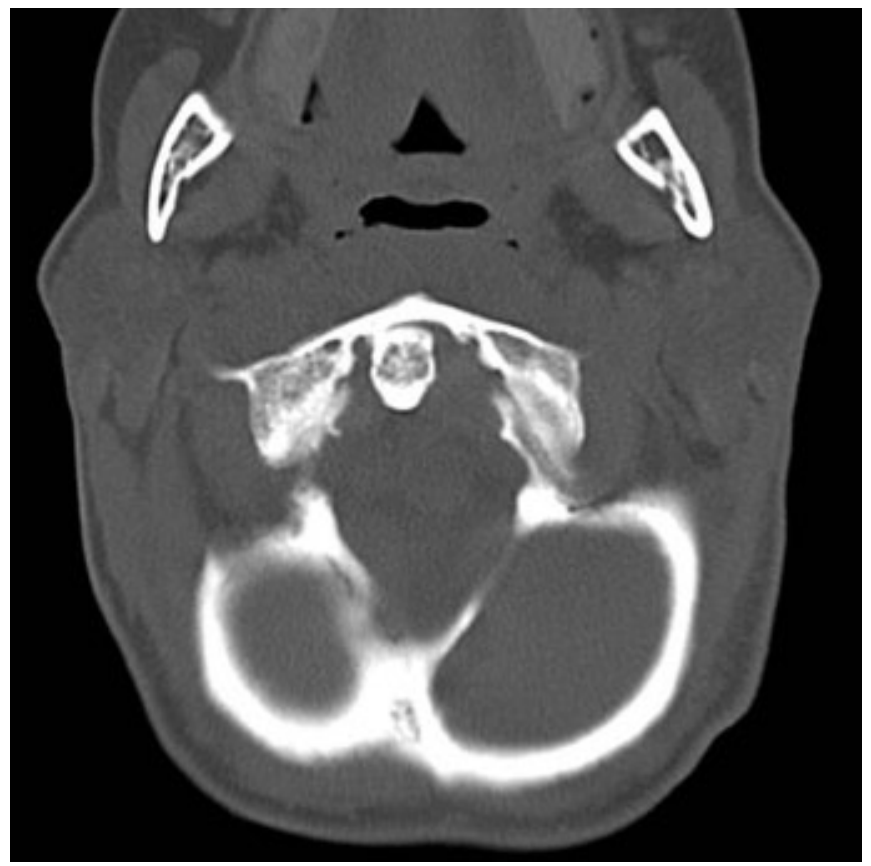

Figure 3: CT-scan. There is no bone decalcification nor rotatory subluxation

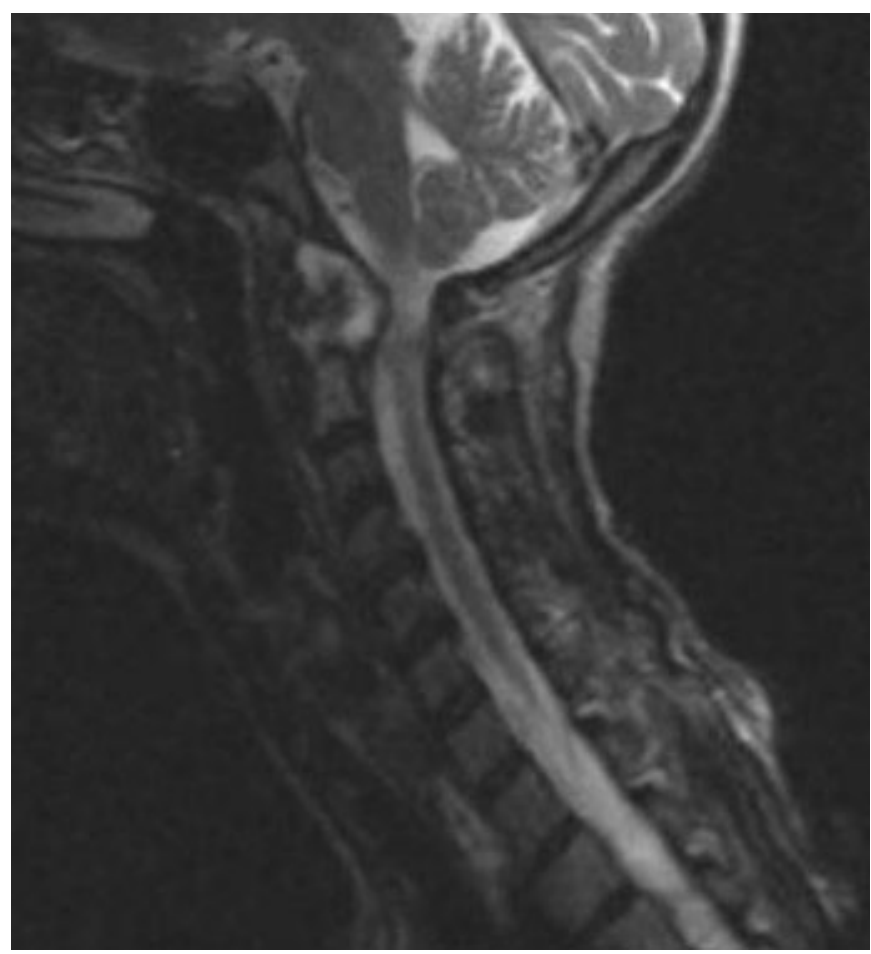

Figure 4: Sagital T2WI before treatment. We see the peri-odontoidal infiltration with compression of the bulbo-medullary junction.

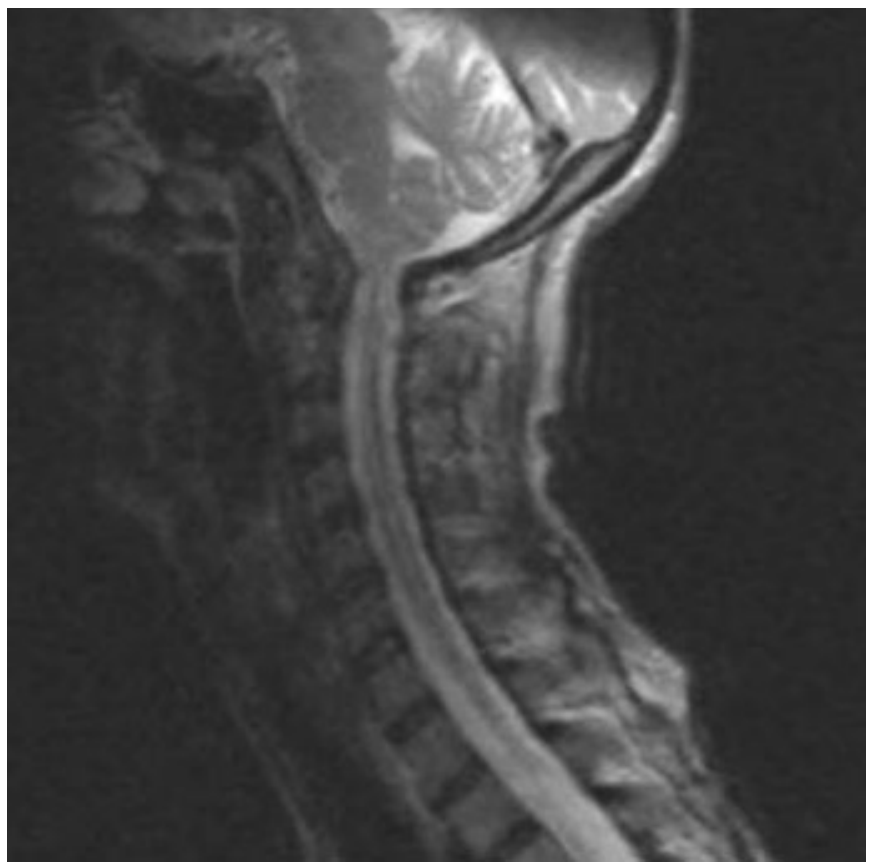

Figure 5: Sagital T2WI after two months of treatment. Resolution of the peri-odontoidal infiltration with decrease of the compression. 


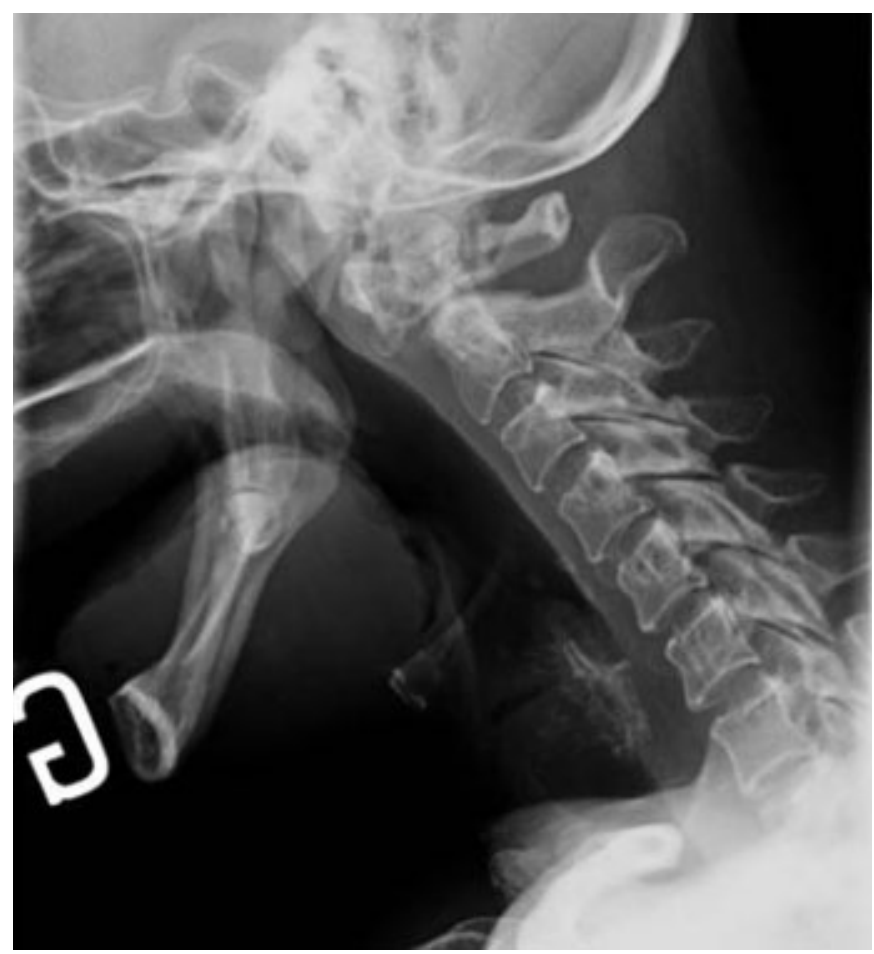

Figure 6: Increased Atlanto dental interval (ADI) in the flexion x-ray. C1-C2 instability.

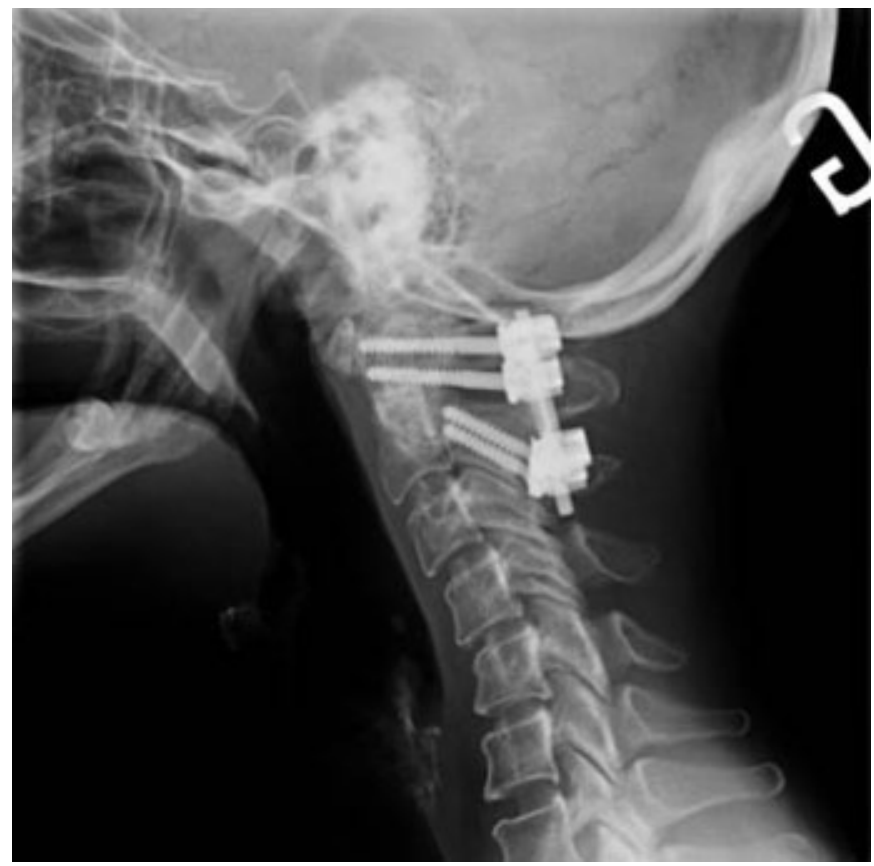

Figure 7: Surgical fixation. We see the reduction of the ADI interval.

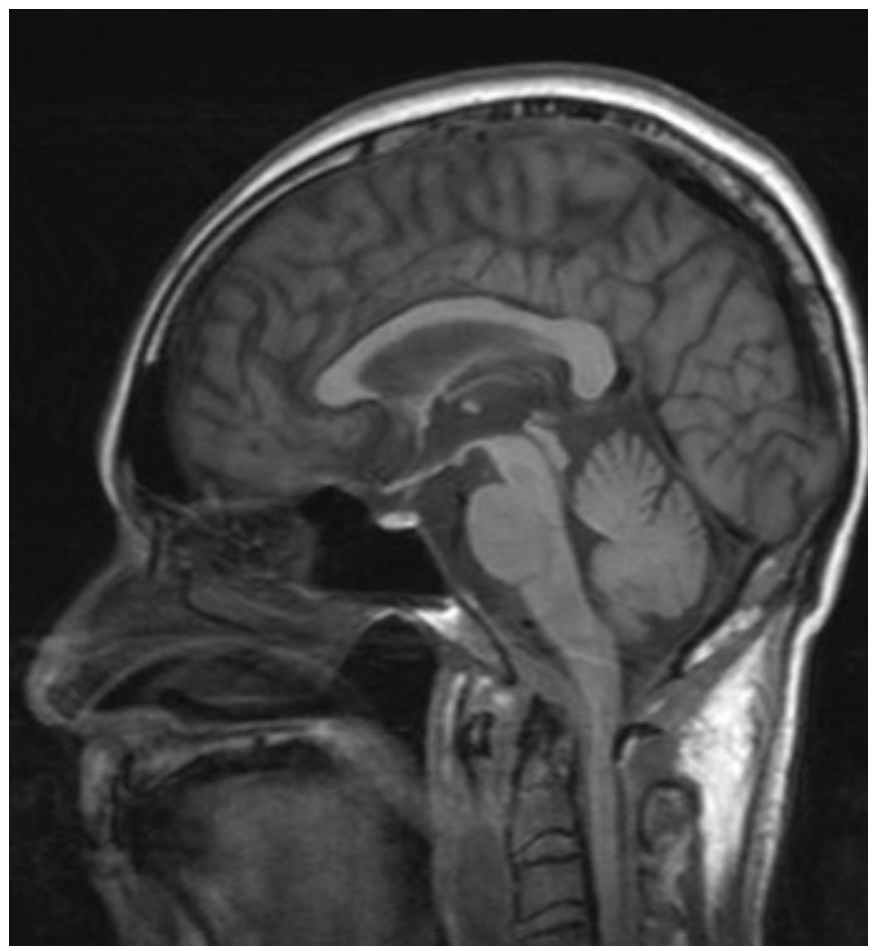

Figure 8: Sagital brain MRI showing the peri-odontoidal infiltration and the increased $A D I$

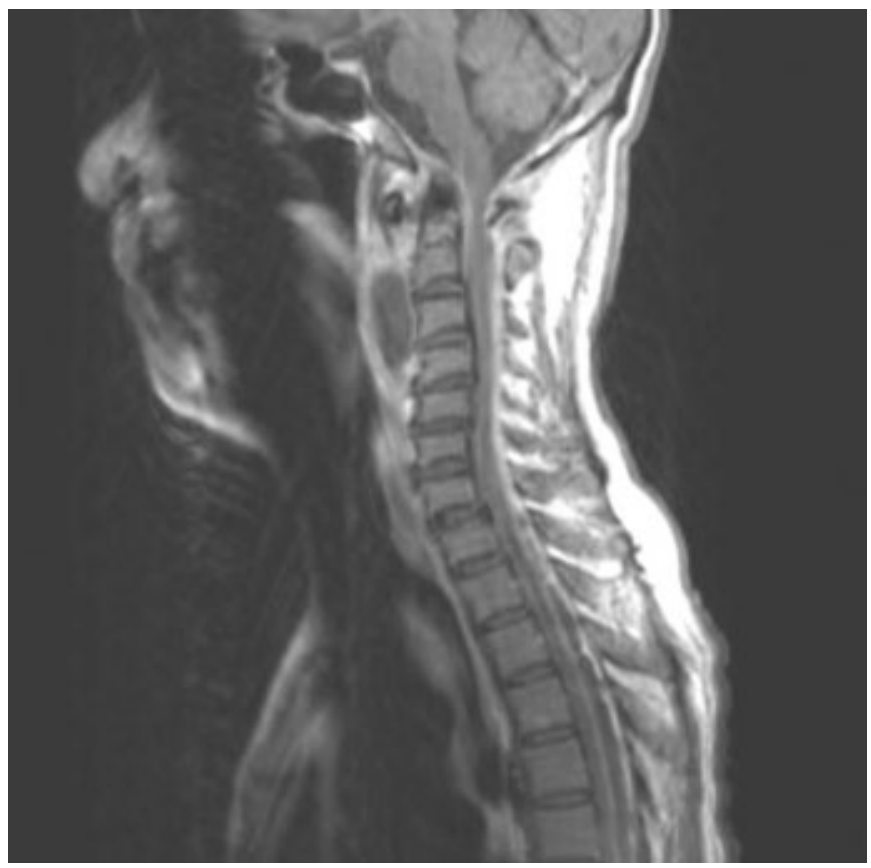

Figure 9: Sagital T1WI with Gado that shows the retropharyngeal abcess with the increased $A D I$. 


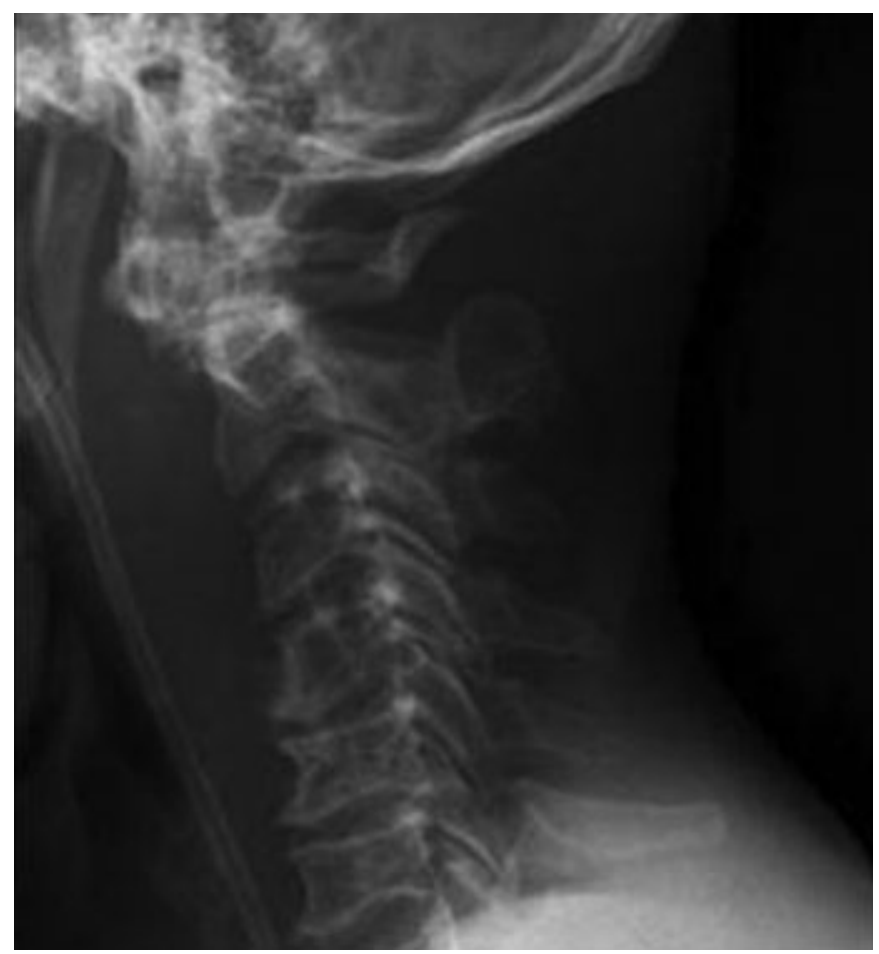

Figure 10: Lateral plain X-ray showing the increased ADI.

(Figure 11) and a transoral evacuation of the retropharyngeal abcess. After surgery the patient completed antibiotic treatment and he was doing well at two months follow-up.

\section{Discussion}

Grisel syndrome was first described in 1830 by Sir Charles Bell $^{2}$ and until now there is controversy regarding the pathogenesis, the diagnosis, and treatment of this syndrome. Grisel reported three cases in 1930 and defined this syndrome as an atlanto-axial instability from inflammatory ligamentous laxity in the head and neck ${ }^{1}$. It is a rare disease and usually affects children, but infrequent adult cases do occur. Several entities were reported in the literature to be associated with this syndrome: pharyngitis, nasopharyngitis, adenotonsillitis, tonsillar abcsess, parotitis, cervical abscess, otitis media ${ }^{1}$, nasopharyngeal operations (velopharyngoplasty) ${ }^{2}$, and after adentonsillectomy with use of monopolar suction cautery ${ }^{3}$. Several theories have been proposed to explain its pathogenesis: 1) by Witteck; metastatic inflammatory effusion cause ligamentous stretching and subluxation, 2) by Grisel; subluxation occurs on the basis of muscular spasm, and 3) the most accepted mechanism by most authors today; hyperemia following infection or trauma that leads to decalcification of the anterior arch of $\mathrm{C} 1$ and laxity of the transverse ligament.

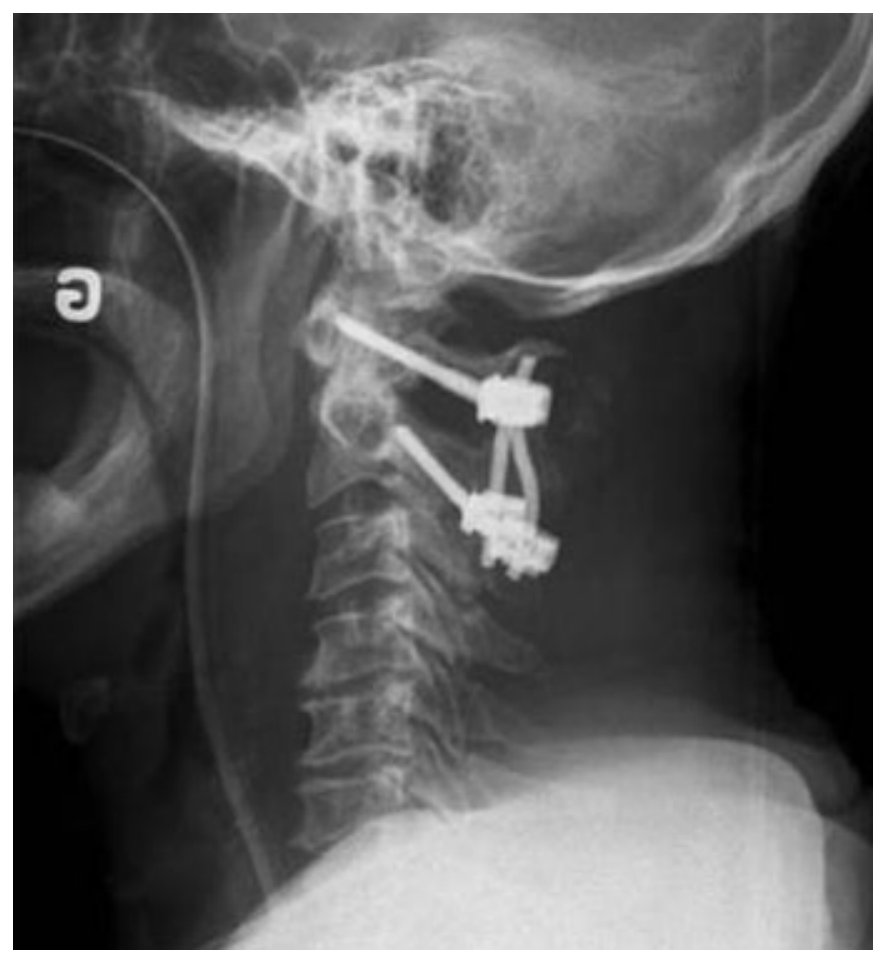

Figure 11: Surgical fixation and reduction of the ADI.

Anatomical findings that support this theory: the peri-odontoidal vascular plexus drains in the postero-superior pharyngeal region ${ }^{1,3,4}$.

This condition is more common in children because the upper spine is more mobile than in adults and they have a hypertrophic status of the peripharyngeal lymphoid tissue which is located in the area drained by the pharyngovertebral plexus ${ }^{2}$.

Clinical manifestations include: neck stiffness, torticollis, neck pain on attempted motion and dysphagia. Neurological complications occur in $15 \%$ of cases and include radiculopathy, quadriplegia and death from cord compression ${ }^{1}$. Clinical signs include: 1) Sudeck's sign where spinous process of $\mathrm{C} 2$ is palpable away from the midline at the site opposite to the dislocation and 2) Cock-Robin sign which is torticollis with the chin turned away from the subluxated side. The head is tilted towards the affected side and flexed anteriorly making it difficult to open the mouth ${ }^{1}$.

Imaging is very important in the diagnosis and follow-up of these patients ${ }^{4}$ : An open-mouth view can provide good visualization regarding rotational deformity. Antero-posterior view can reveal asymmetry between the facet joints. Lateral view reveals an increased atlanto-dental interval and dynamic views show the instability. A CT-scan with $3 \mathrm{D}$ reconstruction offers detailed illustration of the bony cervical spine and 
subluxation. Magnetic resonance imaging is helpful for evaluating the surrounding soft tissue structures as well as the presence of spinal cord compression. The CT-scan and MRI are very helpful and should be done early if there is a suspicion of Grisel syndrome, to avoid delays in the diagnosis.

Fielding has described a working classification of atlantoaxial subluxation into four distinct types ${ }^{6}$. In type I, the atlas is rotated on the odontoid and there is no anterior displacement of the atlas. In type II, the atlas is rotated on one lateral articular process with 3 to $5 \mathrm{~mm}$ of anterior atlas displacement. In type III, there is rotation of the atlas with anterior displacement greater than 5 $\mathrm{mm}$. In type IV, there is rotation and posterior displacement of the atlas. Types III and IV are the least common overall but the most likely to develop spinal cord compression. This classification is valid for all types of atlantoaxial rotatory subluxations, where the Grisel syndrome is the one that follows a respiratory tract or a pharyngeal infection ${ }^{5}$. The treatment is first of all medical (antibiotics if an infection is established). Other treatment modalities (immobilization) depend on the stability of $\mathrm{C} 1 / \mathrm{C} 2$. If the transverse ligament is intact, a cervical immobilization with a hard cervical collar for six to eight weeks is recommended. If the transverse ligament is deficient, the neck should be stabilized by cervical skeletal traction with GardnerWells or immobilized with Minerva cast or halo vest. If the patient has subluxation lasting longer than three months, primary $\mathrm{C} 1 / \mathrm{C} 2$ fusion is recommended ${ }^{1}$.

Our two adult patients presented with Grisel's syndrome. The first after a tonsilopharyngitis and the second after a retropharyngeal abcess. The first presented with Lhermitte's sign and a moderate nuchal rigidity and the second with nuchal rigidity in the context of sepsis and retropharyngeal abcess. In the two cases we had an instability of C1-C2 with deficiency of the transverse ligament, revealed by the increase of the atlantodental interval. The medical treatment alone failed in the first case. The internal fixation reduces the atlanto-dental interval in the two cases and the patients are stabilized.

According to our experience and in the light of the literature :

1) Grisel's syndrome is a rare disease that we should rule out in a patient presenting with neck pain with or without neurological deficit after upper respiratory tract infections.

2) Recognition of Grisel's syndrome and early intervention is a critical factor for a successful outcome.

3) Surgical treatment depends on the grade of the disease, the stability of $\mathrm{C} 1-\mathrm{C} 2$, and the response to medical therapy (antibiotics).

\section{REFERENCES}

1. Ugur HC, Caglar S, Unlu A, Erdem A, Kanpolat Y. Infection-related atlantoaxial subluxation in two adults: Grisel syndrome or not? ActaNeurochir (ien). 2003 Jan;145(1):69-72.

2. Isern AE, Ohlin A, Stromblad LG, Johnell IR, Becker M, Svensson H. Grisel syndrome after velopharyngoplasty. Scand J Plast Reconstr Surg Hand Surg. 2004;38(1):53-7.

3. Martinez-Lage JF, Martinez Perez M, Fernandez Cornejo V, Poza M. Atlanto-axial rotatory subluxation in children: early management. Acta Neurochir (Wien). 2001 Dec; 143(12): 1223-8.

4. Yu KK, White DR, Weissler MC, Pillsburry HC. Nontraumatic atlantoaxial subluxation (Grisel syndrome): a rare complication. Laryngoscope. 2003 Jun;113(6):1047-9.

5. Meek MF, Hermens RAEC, Robinson PH. La maladie de Grisel: spontaneous atlantoaxial subluxation. Cleft Palate Craniofac J. 2001 May;38(3):268-70.

6. Fielding JW, Hawkins RJ, Hensinger RN. Atlanto-axial deformities. Orthop North Am. 1978;9:955-67. 